TECHNICAL REPORT 3006

December 2015

\title{
Near-Field HF Antenna Pattern Measurement Method Using an Antenna Pattern Range
}

Ani Siripuram

Michael Daly

Approved for public release.

SSC Pacific

San Diego, CA 92152-5001 


\title{
SSC Pacific \\ San Diego, California 92152-5001
}

K. J. Rothenhaus, CAPT, USN

Commanding Officer

C. A. Keeney

Executive Director

\begin{abstract}
ADMINISTRATIVE INFORMATION
The work described in this report was performed during Fiscal Year 2015 by the Applied Electromagnetics Branch (Code 52250) of the System of Systems (SoS) \& Platform Design Division (Code 52200), Space and Naval Warfare Systems Center Pacific (SSC Pacific), San Diego, CA. The Naval Innovative Science and Engineering (NISE) Program at SSC Pacific provided funding for this Applied Research project.

Released by

D. E. Hurdsman, Head

Applied Electromagnetics Branch

Under authority of

J. McGee, Head

SoS \& Platform Design

Division
\end{abstract}

This is a work of the United States Government and therefore is not copyrighted. This work may be copied and disseminated without restriction. 


\section{EXECUTIVE SUMMARY}

The Antenna Pattern Range (APR) is an essential measurement facility operated at Space and Naval Warfare Systems Center Pacific (SSC Pacific). One proven capability of the APR is the measurement of far-field antenna patterns intended for placement above a large conducting ground plane. While antenna pattern measurements are traditionally limited to the very high frequency (VHF) to ultra-high frequency (UHF) regime, the SSC Pacific APR is a far-field measurement facility.

Absolute antenna gain is a critical component to any link budget. This report focuses on computing absolute gain for HF antennas measured on the APR. Recent research efforts by SSC Pacific's Applied Electromagnetics Branch (Code 52250) show that the APR extends to accurate measurement of normalized far-field radiation patterns of HF antennas. The normalized pattern measurement is done through a far-field extrapolation of near-field measurements. However, until now, no calibration method existed to determine the absolute gain of an HF antenna under test on the APR.

This report documents work performed in fiscal year 2015 to assess and further develop the APR's HF far-field measurement capabilities. The proposed measurement technique uses both measured quantities and electromagnetic simulation. The primary objective of this report is to document the near-field pattern measurement method and present selected HF radiation patterns extracted from near-field measurements. 


\section{CONTENTS}

EXECUTIVE SUMMARY ...........................................................................................ii

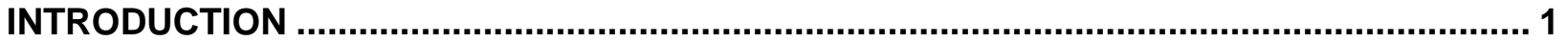

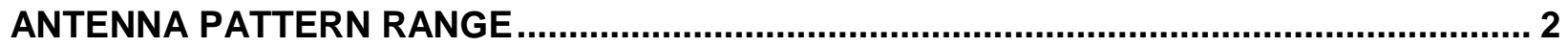

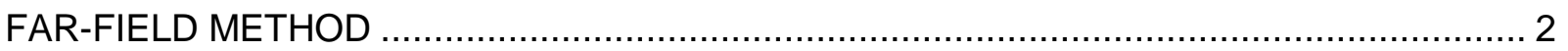

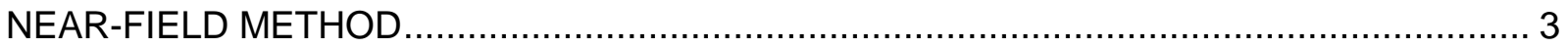

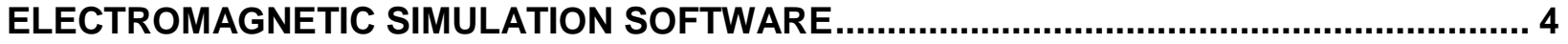

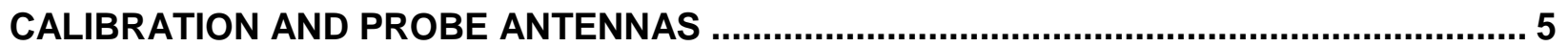

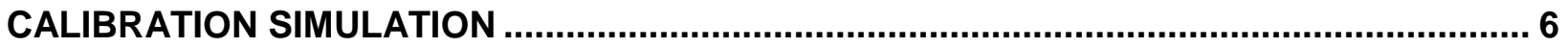

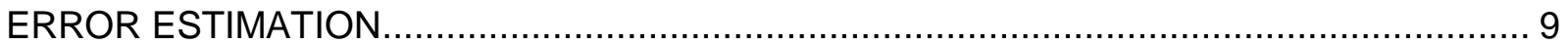

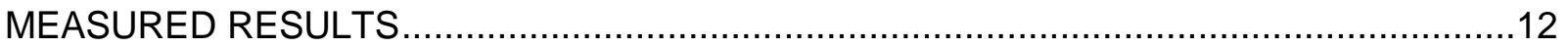

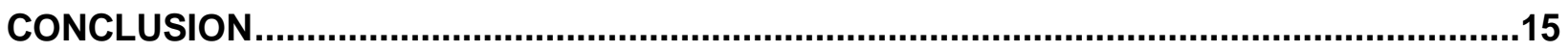

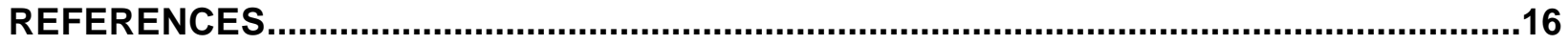

Figures

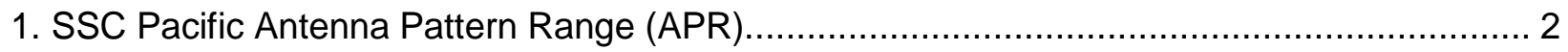

2. Flowchart for directivity (blue) and gain measurement (green) ................................... 4

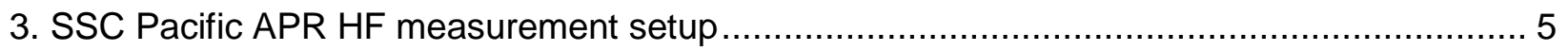

4. Calibration antenna impedance measurement comparison ...................................... 6

5. Probe antenna near-field test scenario...................................................................... 7

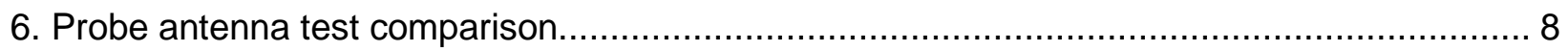

7. Probe antenna near-field test comparison to $45^{\circ}$ elevation reference.............................. 8

8. Relative magnitude and phase difference of calibration to probe antenna coupling for lossy

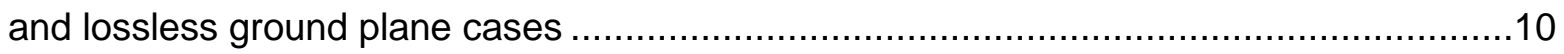

9. Error comparison of far-field electric field at $30 \mathrm{MHz}$ for Hertzian dipole sources ..................11

10. Error comparison of far-field electric field at $16 \mathrm{MHz}$ for Hertzian dipole sources ................12

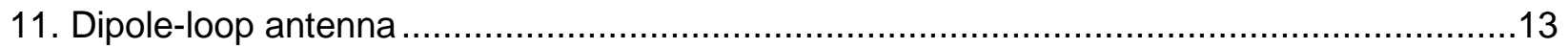

12. Far-field simulation and measurement comparison for dipole-loop antenna at $14 \mathrm{MHz}$.......14

13. Far-field simulation and measurement comparison for dipole-loop antenna at $14 \mathrm{MHz}$ in $\mathrm{dB}$ (power reference) 


\section{INTRODUCTION}

Accurate characterization of antennas designed to support the warfighter is a critical step in a given antenna's development and fielding. Though electromagnetic simulation software can be used as a design tool, measurement of the physical antenna in its operational environment remains the standard for performance verification. The Space and Naval Warfare Systems Center (SSC Pacific) Antenna Pattern Range (APR) can emulate such environments for antenna pattern measurement.

The APR is a far-field measurement facility whose main function is the pattern measurement of scale (smaller) model antennas measured at scaled (higher) frequencies. The size and frequency scaling typically assures that measurement is taken in the far-field of the antenna. In the case of a radiating structure, such as an antenna, the rule-of-thumb for the far-field regions is $R>2 D^{2} / \lambda$ where $R$ is the radial distance from the antenna and $D$ is the largest dimension of the antenna [1] [2]. For an antenna with a $D$ of $10 \mathrm{~m}$ and for $\lambda$ at $10 \mathrm{~m}$ (30 MHz), $R$ is $20 \mathrm{~m}(66 \mathrm{ft})$, which is near the $80-\mathrm{ft}$ radius of the APR. Therefore, using the APR to extract high-frequency (HF) antenna patterns at 2- to $30-\mathrm{MHz}$ frequencies may require near-field measurement technique.

A recent Applied Electromagnetics Branch (Code 52250) HF antenna characterization effort led to the development of a method using near-field APR measurements to compute antenna directivity. Directivity is an essential component of antenna characterization. However, directivity does not include any information about the antenna's radiation efficiency, which is defined as the ratio of the power an antenna accepts to the power it radiates. The product of directivity and radiation efficiency is an antenna's absolute gain. Unlike directivity, absolute gain can be used in link budget calculations. The need for absolute gain motivated development of an accurate way to combine APR measurement and numerical simulation to yield the absolute gain of HF antennas. 


\section{ANTENNA PATTERN RANGE}

\section{FAR-FIELD METHOD}

Figure 1 shows the SSC Pacific APR. The APR consists of a fiberglass tripod arch on top of a conductive ground plane. The ground plane consists of a 12-ft-radius, brass-plated turntable centered in an 80-ft-radius, aluminum-coated concrete slab. The remainder of the ground plane consists of a conductive wire mesh embedded in asphalt that extends well beyond the 80 -ft-radius aluminum ground. The combination of the brass-plated turntable, aluminum-coated concrete, and embedded wire mesh provides a very large and relatively low loss ground plane for an antenna under test (AUT). The fiberglass arch contains a track upon which a probe antenna sweeps through elevation angles. The probe antenna moves along the arch track maintaining a radial distance of approximately $80 \mathrm{ft}$ between the antenna and the center of the turntable between elevation angles of $3^{\circ}$ and $90^{\circ}$. An AUT can be placed in the center of the turntable and "thru" measurements between the AUT and the probe antenna can be made for any desired combination of azimuth and elevation angles along the 80-ft-radius hemisphere.

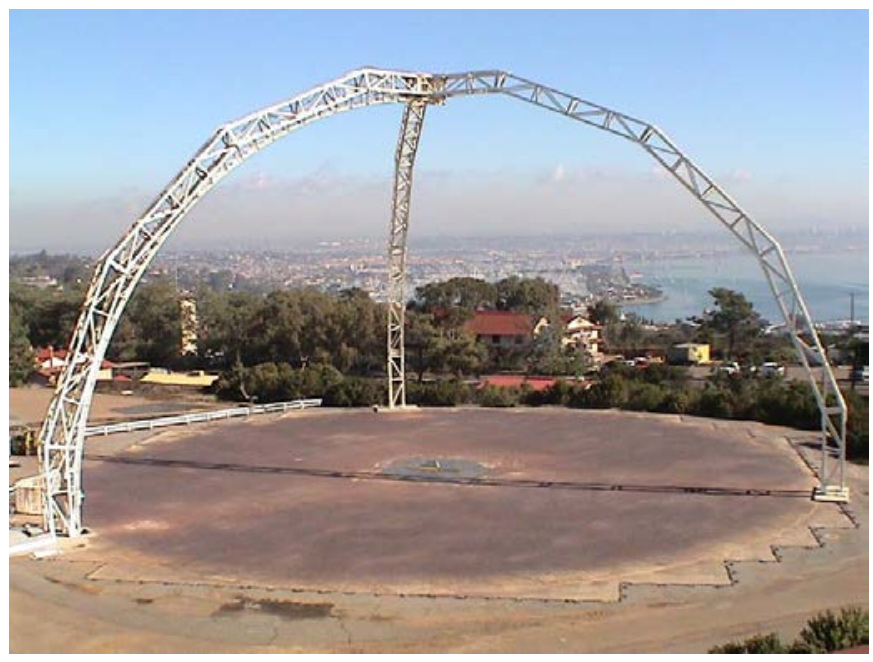

Figure 1. SSC Pacific Antenna Pattern Range (APR).

Using a network analyzer thru measurement and a bank of calibration $1 / 4 \lambda$ monopole antennas that cover the very high frequency/ultra-high frequency (VHF/UHF) measurement range of interest, the APR can be used to extract the gain of an AUT. The expression used to relate AUT measurements taken on the APR to AUT gain as a function of $\varphi$ and $\theta$ is given by Equation (1):

$$
G^{\text {AUT }}(\varphi, \theta)=\frac{\left|S^{\text {AUT/Probe }}(\varphi, \theta)\right|^{2}}{\left|S^{\text {Cal/Probe }}(\varphi, \theta)\right|^{2}} G^{\text {Cal }}(\varphi, \theta)
$$

In Equation (1), $S_{A U T / P r o b e}$ is the $S$-parameter coupling between the AUT and the probe antenna, $S_{\text {Cal/Probe }}$ is the $S$-parameter coupling between a given calibration antenna and the same probe antenna, and $G^{\mathrm{Cal}}$ is the known gain of the calibration antenna. The APR measurement point for $\theta=0^{\circ}$ corresponds to the probe antenna track position directly above the center of the turntable. Since the turntable can rotate $360^{\circ}$ in azimuth, a $\varphi=0^{\circ}$ position can be defined arbitrarily. 
The measured quantities in Equation (1) do not require any network analyzer calibration since the only difference between the two necessary measurements is the replacement of the calibration antenna with the AUT. In the calibration discussed in this report, the calibration antenna's gain is found through simulation. The simulation must be sufficiently accurate such that it includes all significant physical aspects of the antenna itself and all effects of the APR's ground plane (i.e., ohmic loss). The electromagnetic effects of the fiberglass arch are typically ignored in the simulation. For the VHF and UHF regimes, the APR calibration antennas are simple wire monopoles that can easily modeled with available simulation software. To extend the APR's use to the HF regime, HF calibration antennas must be used. However, some of the assumptions made for VHF and UHF antennas may not hold for HF antennas. A probe antenna at a radius of $80 \mathrm{ft}$ will not typically be in the far-field of an HF AUT. Additionally, the calibration antenna may have to be modeled with a significant amount of detail to capture its near-field interaction with the ground and with the probe antenna. The specific material composition of the ground plane (brass, copper, etc.) is typically not included for VHF/UHF simulations but will be included for the HF simulation.

\section{NEAR-FIELD METHOD}

If the AUT and probe antennas are in each other's near-field, a simple relationship between the measured $S$-parameter and a desired far-field quantity, such as gain, may not exist. A pattern extraction technique that utilizes near-field measurement requires additional consideration.

One consideration is that the probe antenna should be chosen such that it does not significantly alter the field radiated by the AUT. Specifically, the probe antenna's physical presence should not significantly alter the field value it is used to measure. In the case of a far-field measurement, the probe antenna doesn't alter the field it is intended to probe because its physical dimensions are typically much smaller than the radial distance to the AUT. Though a probe antenna small enough to fit on the arch track is significantly smaller than $80 \mathrm{ft}$ (the distance to the center of the turntable), one must verify whether such a probe is sufficiently non-invasive for the APR must be verified.

Once an accurate means of measuring the near-field field value is available, this near-field measurement must be transformed into the far-field. The transformation of a near-field measurement into the far-field can be done using the Equivalence Theorem that is summarized as follows: knowledge of the tangential component of the electric or magnetic field on a closed surface surrounding any source implies knowledge of the unique field radiated by that source [3]. In case of the APR measurement, the closed surface is an 80 - $\mathrm{ft}$ hemisphere upon which the probe antenna can take measurements and the enclosed source is the AUT.

Unfortunately, the equivalence principle assumes that one has access to an infinite number of field points on that enclosing surface. Actual measurements cover only a finite number of field test points. Therefore, an accurate near-to-far field extraction method using these discrete field test points must be employed. A tested code doing just this was developed by Code 52250 in fiscal year (FY) 2013 and FY14 [4]. The code uses an alternative spherical harmonic expansion method of Sarkar, Petre, Taaghol, and Harrington [5].

The near-to-far field extraction code requires an accurate representation of the tangential field (electric or magnetic) at a finite number of points on the APR hemisphere. Therefore, a near-field pattern extraction technique requires (1) that the thru measurement between the calibration antenna and the probe antenna is a non-invasive representation of the tangential field quantity at all measurement points on the APR hemisphere, and (2) an accurate relationship between the thru measurement and the actual tangential field on the APR hemisphere is known. We propose that 
(1) and (2) can be verified and satisfied, respectively, through electromagnetic simulation of the calibration and probe antennas.

A flowchart of the existing APR HF antenna directivity measurement method (top) and the proposed APR HF antenna gain measurement method (bottom) are shown in Figure 2. The two methods are susceptible to the same type of measurement error and near-to-far field transformation error. Contributors to measurement error include environmental factors such as scattering objects near the APR (cars, buildings, etc.), inadvertent reception (spurious HF signals, radiating antenna cables), or insufficient signal-noise ratio (SNR). Reflections from objects near the APR are suppressed in measurements via time-gating. The near-to-far field transformation error is a consequence of the mathematical extrapolation of a finite number of near-field measurement points into a far-field quantity. Since the proposed gain measurement method requires calibration through simulation, any simulation error will translate into error in the computed gain. The sources of error will be discussed in more detail in the Error Estimation section of this report.

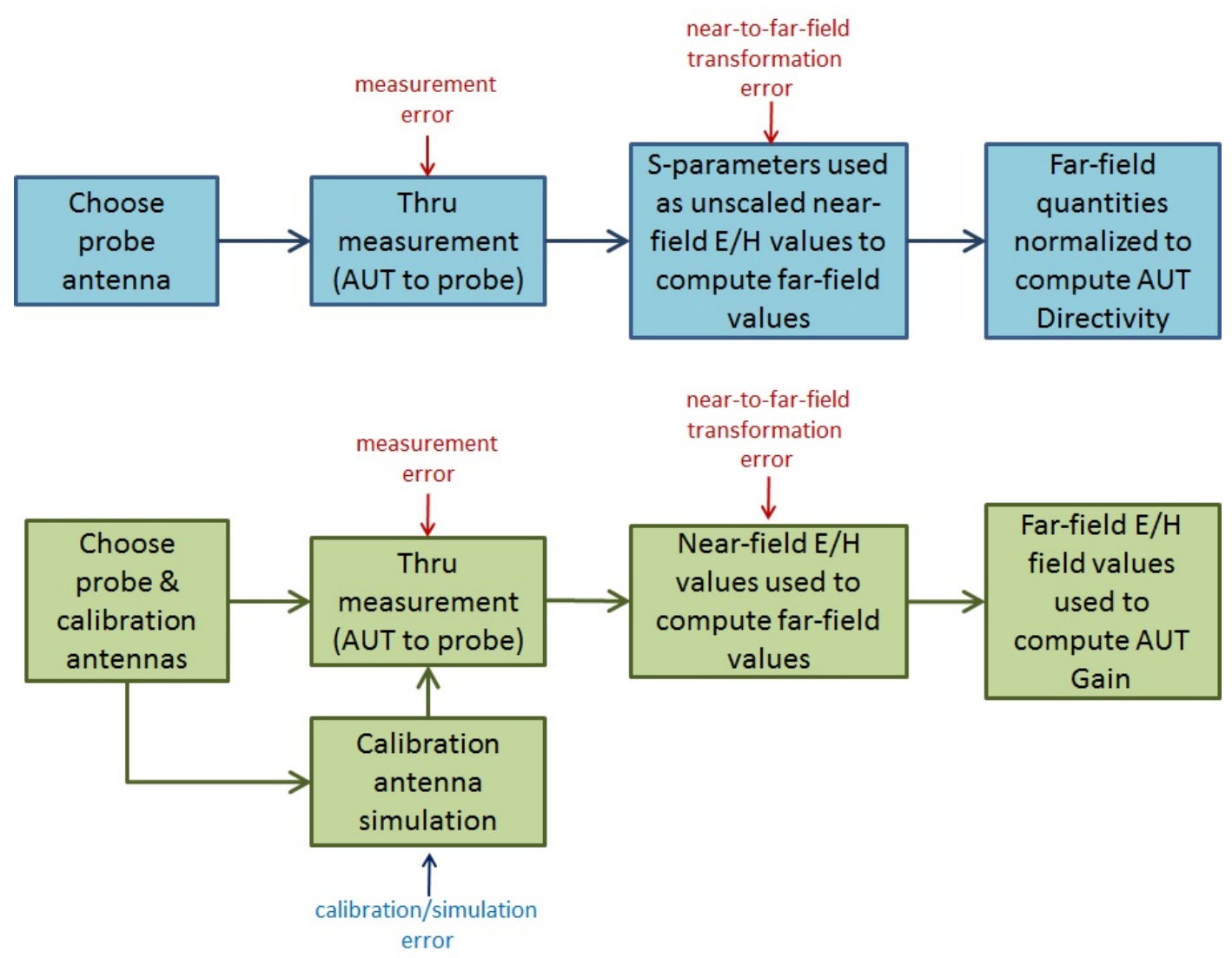

Figure 2. Flowchart for directivity (blue) and gain measurement (green).

\section{ELECTROMAGNETIC SIMULATION SOFTWARE}

The computational software used must accurately model the physical details of a potentially large and intricate calibration antenna. The software must also allow for the inclusion of all significant features of the APR. The APR consists of a brass turntable surrounded by an aluminum-coated ground plane and a large conductive ground mesh. FEKO is a commercial electromagnetic simulation software that satisfies such requirements [6]. FEKO enables designers to model complex three-dimensional (3D) structures and include ohmic losses in a ground plane. Designers can use 
FEKO to individually model the three principle features of the simulation model (calibration antenna, probe antenna, and ground plane) with a high degree of accuracy.

\section{CALIBRATION AND PROBE ANTENNAS}

The probe antenna used in the prior directivity measurement effort was an 86" dipole antenna with a balun. The dipole antenna is sub-resonant at $30 \mathrm{MHz}$, which means it will exhibit no undesired pattern nulls and the antenna is easy to rotate by $90^{\circ}$ so both electric field polarizations can be measured. The antenna is electrically small enough, even at the higher frequencies, to be considered a relatively non-invasive probe. The only disadvantage is its high mismatch loss over most of the HF band. Fortunately, one can compensate by using an amplifier in the measurement chain to ensure that the calibration antenna is well-matched across the HF band.

Unlike the many resonant calibration antennas used for the APR's VHF/UHF far-field pattern measurement, one calibration antenna will be used for the entire HF band. This simplification is significant because HF antennas are typically large, unwieldy, and expensive. The largest HF antenna available to Code 52250 and that can be placed on the APR turntable is a 35-ft monopole whip (Valcom model AS-2537A). The antenna is relatively well-matched through most of the HF band. A line amplifier can compensate for any significant mismatch at the lower frequencies. The whip passes through an additional resonance around $14 \mathrm{MHz}$, but field calibration points can be chosen away from pattern nulls, if needed. A diagram of the measurement setup is shown in Figure 3.

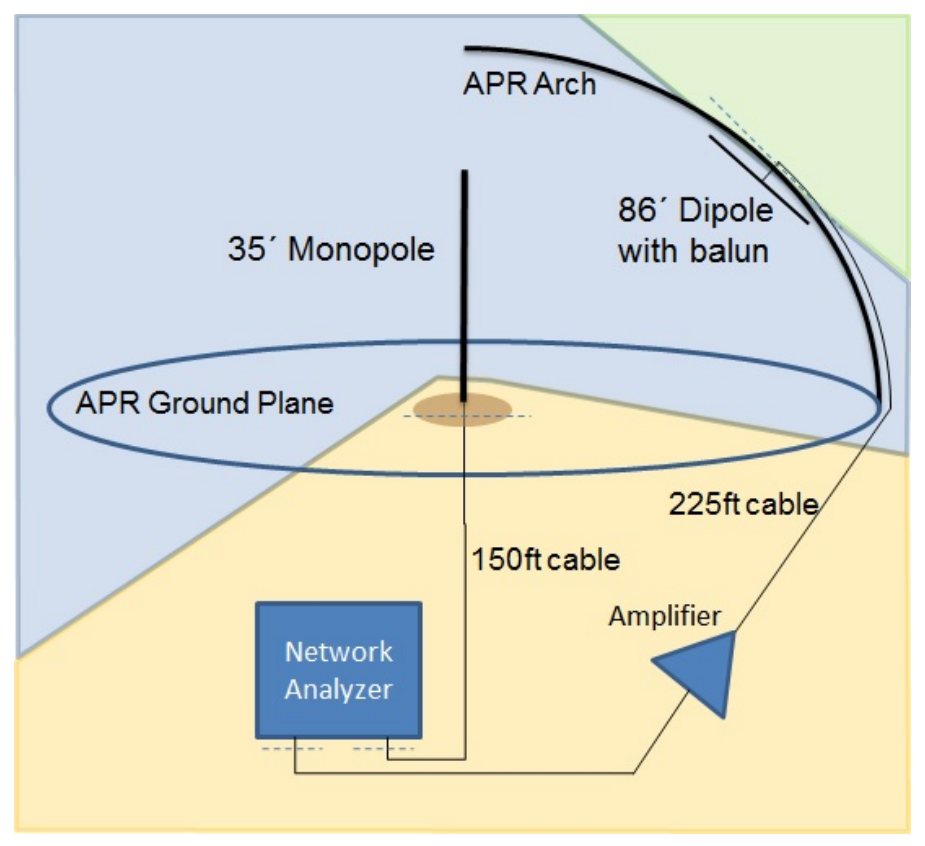

Figure 3. SSC Pacific APR HF measurement setup.

The orange section of Figure 3 covers the amplifier and the feed lines. This portion of the measurement setup remains unchanged for all measurements. The green section covers the transmission line feed and the balun of the probe dipole antenna. In general, factors like the position of the dipole to the ground plane, or the relative location of the dipole in the near-field of an antenna, affect the effective impedance of the dipole. The assumption for this region is that the effective impedance of the dipole feed is invariant to these factors. The near-field interaction is given in blue. 
An assumption that will be made for this region is that calibration based on a vertically polarized calibration antenna (e.g., a monopole) is applicable to the gain measurement of both verticallypolarized and horizontally-polarized AUTs.

\section{CALIBRATION SIMULATION}

In the FEKO simulation, the brass turntable and the aluminum-coated concrete are included, while the embedded mesh portion is assumed to be a perfectly conducting infinite ground plane. This assumption is reasonable because the network analyzer's time-gating feature allows for the gating of potential scatterers outside the 80 -ft radius (e.g., buildings, vehicles, etc.). Therefore, the measurement will not necessarily "see" portions of the ground plane significantly outside the $80-\mathrm{ft}$ radius.

SSC Pacific researchers measured and simulated the 35-ft monopole with a high degree of detail. The dipole antenna is modeled without the balun and twin-line feed. If the effective impedance of the combination of the balun and feed are invariant, then this portion of the dipole can be "absorbed" into the calibration.

The measured and simulated input impedance of the 35-ft monopole, real and reactive components, are compared in Figure 4. The plots reveal some shift in resonance and slightly differing impedance values at the higher frequencies. The differences are possibly a consequence of not modeling the antenna feed or material properties accurately. Exact agreement between the physical and simulated input impedance is not strictly necessary because the proposed calibration method relies on a thru measurement rather than a reflection measurement. What is necessary is that the nearfields of the physical and simulated antennas are identical for the case that both antennas are impedance matched. Input impedance agreement alone cannot confirm that the two near-fields are in agreement. A possible means of estimating error between the simulated and physical calibration antennas is discussed in the Error Estimation section of this report.
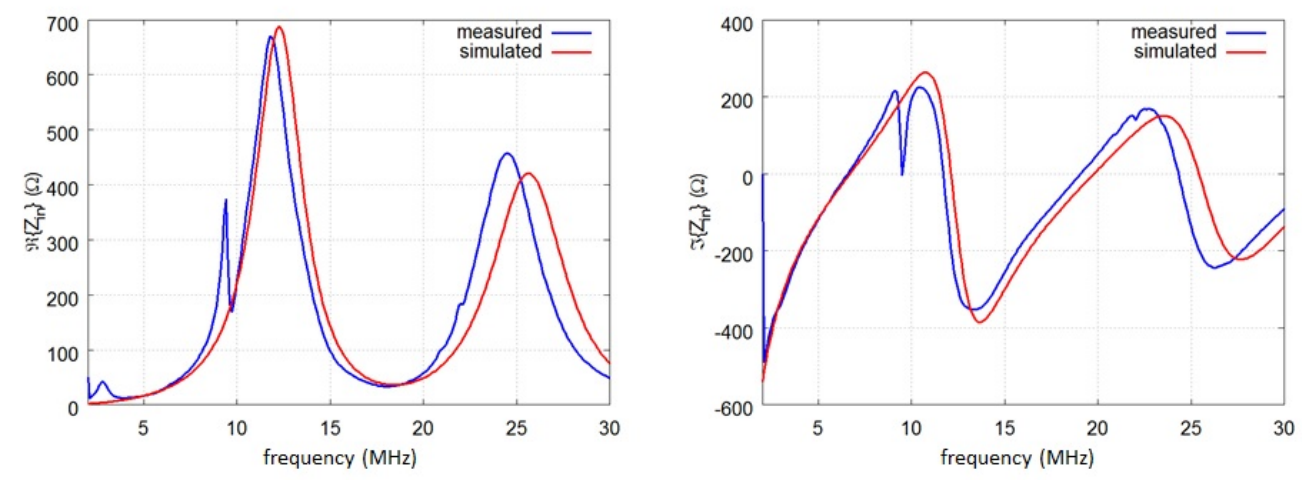

Figure 4. Calibration antenna impedance measurement comparison.

A simple simulation scenario can be used to evaluate the invasiveness of the probe antenna on the electric field radiated by the calibration antenna. First, one should compute the electric field, $E_{\theta}{ }^{n f}$, radiated by the 3-ft calibration monopole at any given point on the APR hemisphere, assuming the 86 " dipole probe antenna is absent. The thru coupling, $S^{n f}$ thru , between the calibration antenna and the probe antenna for the same point on the APR hemisphere can then be computed. Computation of the electric field and thru coupling can be repeated for a point at the same $\theta$ and $\varphi$ angles as the near-field computation, but in the far-field of the calibration antenna as is shown in Figure 5. The superscript 
"ff" denotes a radial distance of $240 \mathrm{~m}$, which is roughly 10 times larger than the $80-\mathrm{ft}$ (24-m) radius of the APR hemisphere. If the ratio of the two near-field quantities and the ratio of the two far-field quantities are identical, then the 86 " dipole should be a sufficiently non-invasive probe at a radial distance of $80 \mathrm{ft}$ from the 35-ft calibration monopole.

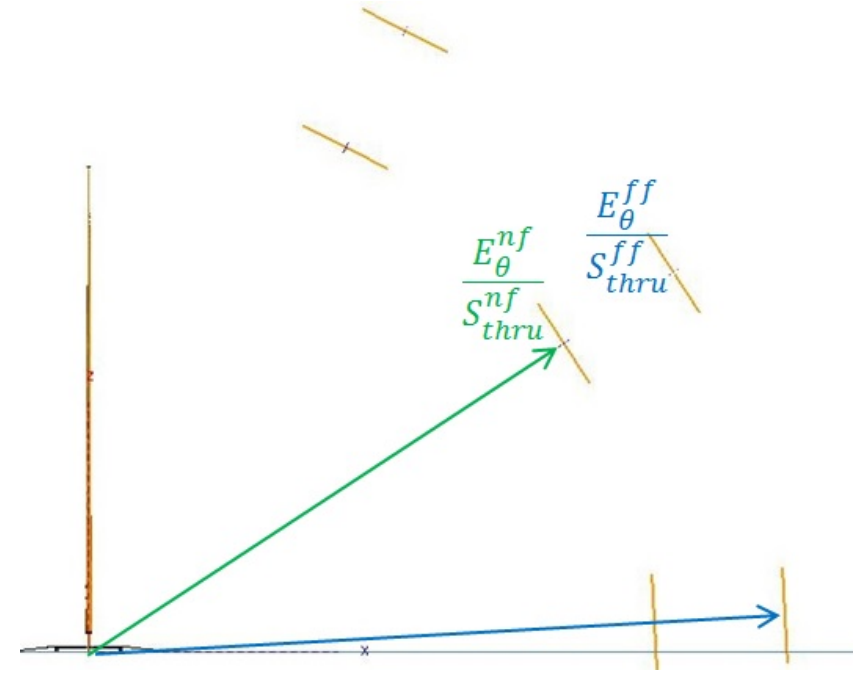

Figure 5. Probe antenna near-field test scenario.

If the two ratios shown in Figure 5 are the same, then one should have a high degree of confidence that the dipole is a good non-invasive probe for the calibration antenna at a distance of $80 \mathrm{ft}$. This test can be done for a number of representative (low, medium, high) elevation angles. Magnitude and phase comparisons at three angles are shown in Figure 6. The magnitudes appear to be in good agreement for all three angles and the phases start to deviate in the upper HF range. A comparison of the magnitude and phase of the three near-field ratios shown in Figure 6 to the near-field ratio at a $45^{\circ}$ elevation is given in Figure 7 . The figures reveal an asymmetric deviation over elevation angle and a general increase in the magnitude and phase deviations as frequency increases. These observations are likely a consequence of interaction with the ground plane.

One reason the 35 - $\mathrm{ft}$ monopole was chosen was because its largest dimension (where $D=70 \mathrm{ft}$ due to the image effect of the conducting ground plane) is near the limit of what can safely be used on the APR. If the probe antenna is sufficiently non-invasive for such an antenna, the same probe antenna should be as equally non-invasive for a physically smaller HF AUT. 

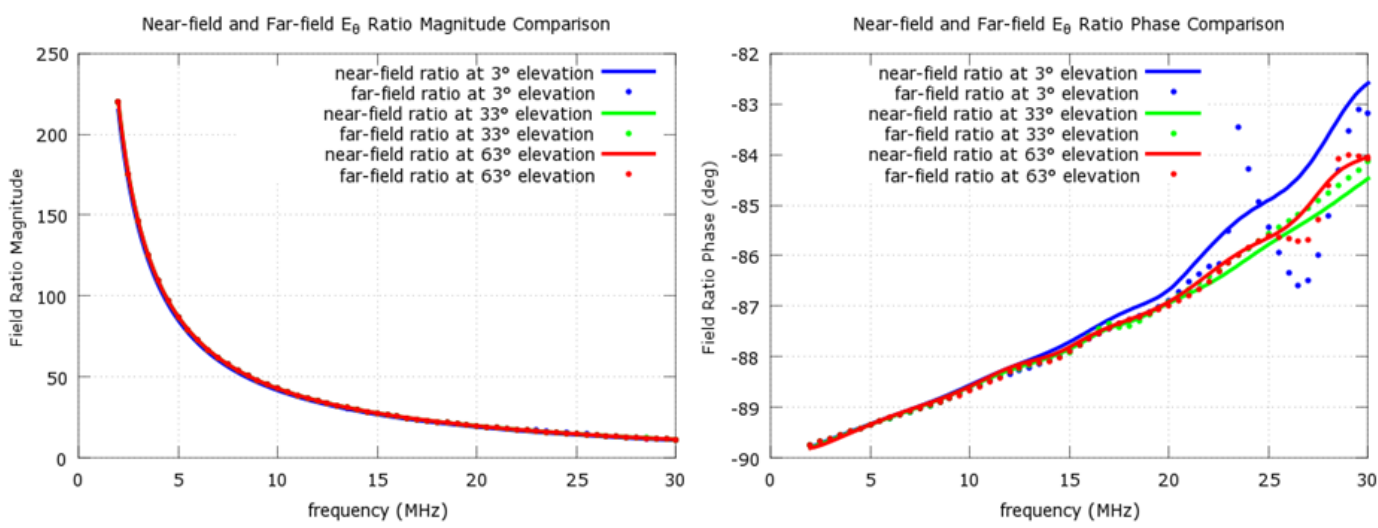

Figure 6. Probe antenna test comparison.

A reason the 35 - $\mathrm{ft}$ monopole was chosen was because its largest dimension (where $D=70 \mathrm{ft}$ due to the image effect of the conducting ground plane) is near the limit of what can safely be used on the APR. If the probe antenna is sufficiently non-invasive for such an antenna, the same probe antenna should be as equally non-invasive for a physically smaller HF AUT.
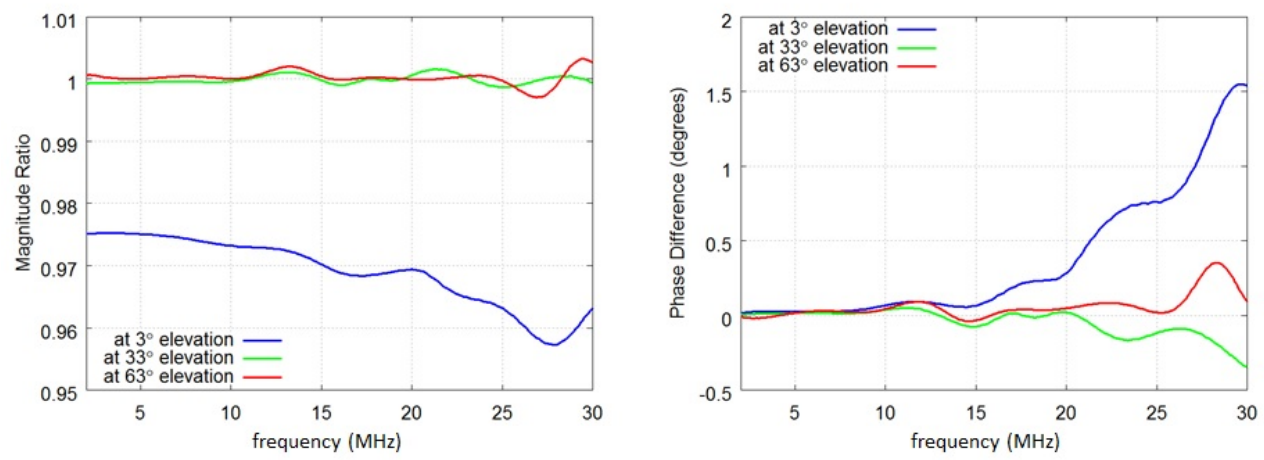

Figure 7. Probe antenna near-field test comparison to $45^{\circ}$ elevation reference.

What remains of the gain measurement method description is how to extract near-field field data from an $S$-parameter (i.e., the thru measurements) measurement. Because the field-to-thru ratios shown in Figure 6 are very similar for the three angles shown, it is reasonable to assume that calibrating based solely on elevation angle measurement simplifies the field extraction process while losing little in accuracy. The expression used to calculate the near-field electric field is given by Equation (2):

$$
E_{\theta}^{A U T}(\varphi, \theta)_{R_{o}}=E_{\theta}^{\text {Cal,sim }} \frac{S_{\text {THRU, }, \theta}^{\text {AUT, meas }}(\varphi, \theta)}{S_{\text {THRU }, \theta}^{\text {Cal,meas }}},
$$

where the left-hand side (LHS) terms represent the electric field component for the AUT at $R_{o}=80 \mathrm{ft}$, the numerator and denominator terms in the right-hand side (RHS) are the thru measurement for the AUT and the calibration antenna, respectively. The AUT measurement is taken at all desired field points on the APR hemisphere while the calibration measurement is only taken at $0^{\circ}$ azimuth and $45^{\circ}$ elevation. The electric field term in the RHS is the electric field at $0^{\circ}$ azimuth and $45^{\circ}$ elevation at a radial distance of $80 \mathrm{ft}$, resulting from simulation of the calibration antenna 
where the antenna accepts $1 \mathrm{~W}$ of power. The consequence is that the computed electric field for the AUT will also be normalized to 1-W power acceptance. Once the tangential electric field on the APR hemisphere is known, it can be fed into the near-to-far field code. The code returns the far-field field values that are then used to compute far-field antenna characteristics such as gain and directivity. For completeness, the equation for the horizontally polarized field is given by Equation (3):

$$
E_{\varphi}^{A U T}(\varphi, \theta)_{R_{o}}=E_{\theta}^{\text {Cal,sim }} \frac{S_{\text {THRU, }, \varphi}^{\text {AUT, meas }}(\varphi, \theta)}{S_{\text {THRU }, \theta}^{\text {Cal,meas }}},
$$

which shows that the vertical calibration ratio is used.

\section{ERROR ESTIMATION}

An estimate of the accuracy of this absolute gain calibration is best done by decomposing the expression of Equation (2) into its constituent parts.

The expression in Equation (2) can be rewritten as shown in Equation (4):

$$
E_{\theta}^{A U T}(\varphi, \theta)_{R_{o}}=S_{T H R U, \theta}^{A U T, \text { meas }}(\varphi, \theta) \frac{E_{\theta}^{\text {Cal,sim }}}{S_{\text {THRU, }, \theta}^{\text {Cal }, \text { sim }}} \frac{S_{\text {THRU }, \theta}^{\text {Cal,sim }}}{S_{\text {THRU }, \theta}^{\text {Cal,meas }}} .
$$

The first term on the RHS is a network analyzer measurement. Although there are errors associated with this term, we assume that sufficient averaging, time-gating, and signal amplification are done such that the measurement error is negligible. The middle term is the calibration term. Error that this term introduces is predominantly associated with the use of a single calibration point $\left(0^{\circ}\right.$ azimuth and $45^{\circ}$ elevation) for all desired measurement points on the APR hemisphere. Designers can use the results (such as those shown in Figure 6) to estimate this error. For example, the phase values at all three elevation angles, over the entire frequency band of interest, fall within $\pm 2^{\circ}$. This $\pm 2^{\circ}$ can be used as an estimate of the phase error for such a calibration. A similar error bounds can be ascribed to the magnitude.

The error associated with last term in the RHS of Equation (3) is a consequence of the inaccuracies in the simulation model. Unfortunately, quantifying this error is difficult because many factors could contribute to differences in the actual thru measurement and the simulated thru measurement. As mentioned in the Calibration and Probe Antennas section of this report, the simulation model neglects the dipole antenna's feed portion and balun under the assumption that the impedance effect of these features is independent of the location of the probe antenna. The feed and balun should have no effect on calibration, as long as this impedance is invariant to measurement location.

One simple way to make an error estimate is to modify the environmental parameter that is presumed to most significantly contribute to simulation error and observe the perturbation in the computed near-field quantities. Other than errors in representing the calibration antenna's feed mechanism, which should not play a significant a role in a thru measurement, we presume that inaccuracies in the representation of the ground plane are the dominant contributors to simulation errors. Therefore, comparison of the calibration-to-probe antenna simulation using the described lossy ground plane and an ideal lossless ground plane may provide an estimate on the sensitivity, and subsequently, the accuracy of the simulation. The plots in Figure 8 show the relative difference between the magnitude and phase of the coupling between the calibration monopole and the probe dipole between the lossy and lossless ground plane simulations. The differences between the two 
simulations can be representative of bounds for the simulation error. For the scenario described, the magnitudes fall within $\pm 2 \%$ and the phases within $\pm 3^{\circ}$.
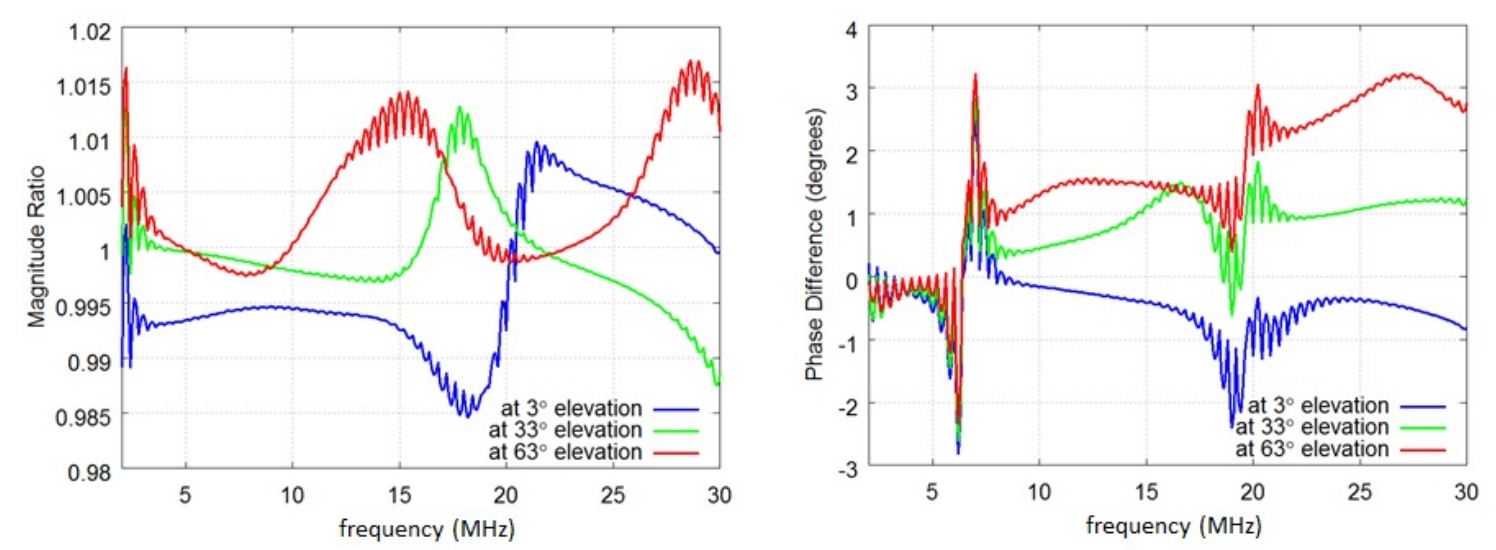

Figure 8. Relative magnitude and phase difference of calibration to probe antenna coupling for lossy and lossless ground plane cases.

Now that error estimates for the each of the three terms in the RHS of Equation (3) can be determined, a method of applying these errors in evaluating their effect on the accuracy of the extracted far-field values can be implemented. This is done using a simulation of Hertzian vertical electric and magnetic dipoles over an infinite perfectly conducting ground plane as the AUT. The two Hertzian dipoles are chosen because together they radiate both vertically and horizontally polarized fields and are easy to simulate using FEKO. Both sources are scaled such that a dramatic difference does not exist between the power radiated in either of the two polarizations. Both Hertzian sources are included in a single simulation. The two estimated errors, which are represented as two operators in Equation (5), introduce the errors in both magnitude and phase. As given in Equation (5) a new error inclusive near-field quantity can be computed and then used in the near-to-far-field extraction code to compute the far-field values. These far-field values can then be compared to the simulated far-field values to assess how the estimated calibration, $e_{1}$, and simulation, $e_{2}$, errors propagate through the near-to-far field extraction. Since the two polarizations are extracted separately, the subscript " $\theta / \varphi$ ” denotes either $\theta$ or $\varphi$ polarization.

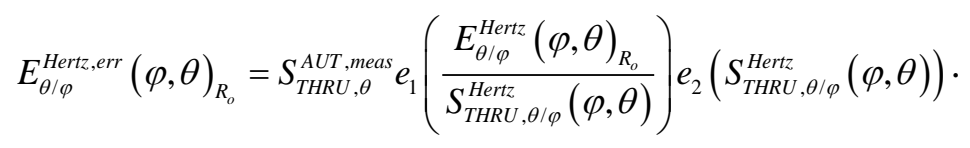

The calibration and simulation error estimates can be made from plots such as those in Figure 7 and Figure 8. Here, $e_{1}$ can introduce magnitude and phase errors of [ $-4 \%$ to $\left.1 \%\right]$ and [ $-0.5^{\circ}$ to $1.5^{\circ}$, respectively, and $e_{2}$ can introduce magnitude and phase errors of $\pm 2 \%$ and $\pm 3^{\circ}$, respectively. Though the error appears to be less significant at the lower frequencies, it is simpler to assign one error value for the entire frequency range with the understanding that the accuracy of the measurement technique might be somewhat better at the lower HF range than the error estimate might indicate. Because the APR and the calibration antenna are relatively rotationally symmetric, the calibration and simulation error estimates are not expected to vary in azimuth.

The error-inclusive field values can be used in the near-to-far field extraction code, yielding the far-field values that can then be compared to the known simulated values. The plots in Figure 9 show the $\theta$ and $\varphi$ directed far-field electric field magnitude at $30 \mathrm{MHz}$ for all 16 possible extremum combinations (plotted in black) of the $e_{1}$ and $e_{2}$ errors. For example, $e_{1}$ errors of $2 \%$ and $-3^{\circ}$ and 
$e_{2}$ errors of $1 \%$ and $-0.5^{\circ}$ in magnitude and phase is one possible combination. A single azimuth angle cut is necessary because the radiated field is invariant in azimuth. The simulated magnitude of the field is plotted in blue.
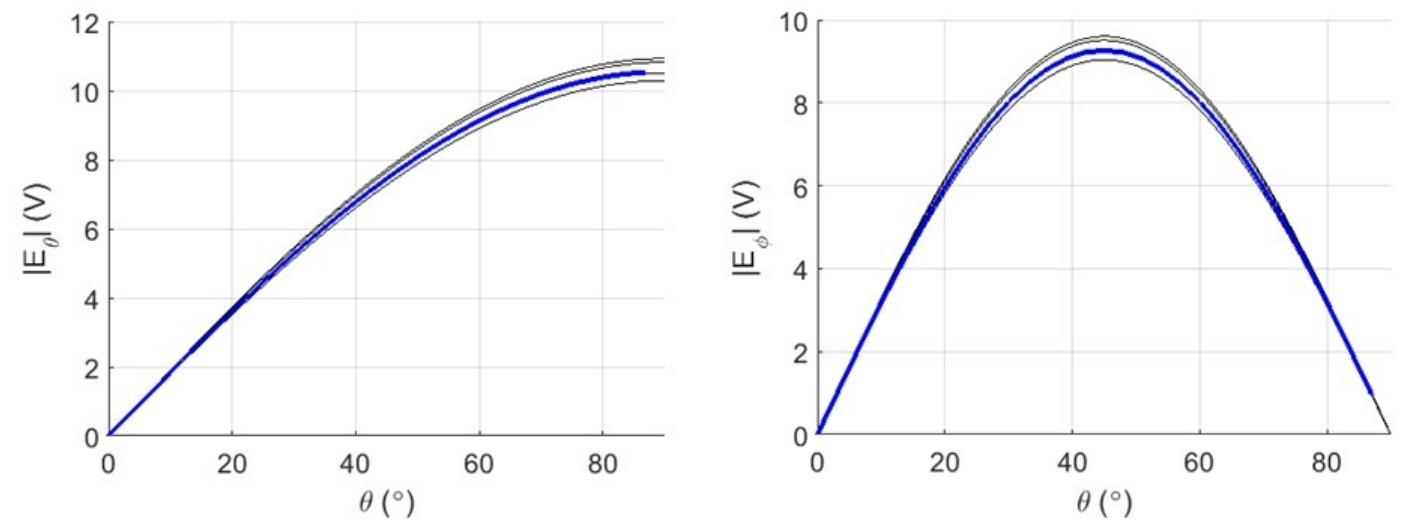

Figure 9. Error comparison of far-field electric field at $30 \mathrm{MHz}$ for Hertzian dipole sources. 
The results in Figure 9 reveal a very symmetric magnitude error for both polarizations of approximately $\pm 5 \%$ or $\pm 0.5 \mathrm{~dB}$ in quantities proportional to power such as gain and directivity. Since the near-to-far field transformation uses only a finite number of test points on the hemisphere, the transformation may be more susceptible to error where there is dramatic variation in the near-field. For example, in the case of the Hertzian magnetic dipole over an infinite ground plane, the source responsible for the $\varphi$-directed electric field shown in Figure 9, there is a dramatic variation of the $\varphi$ component of the near-field (and far-field) electric field as the ground plane is approached. One can observe the effect described by observing the far-field results at a different frequency. Plots of the far-field electric field at $16 \mathrm{MHz}$ are shown in Figure 10.
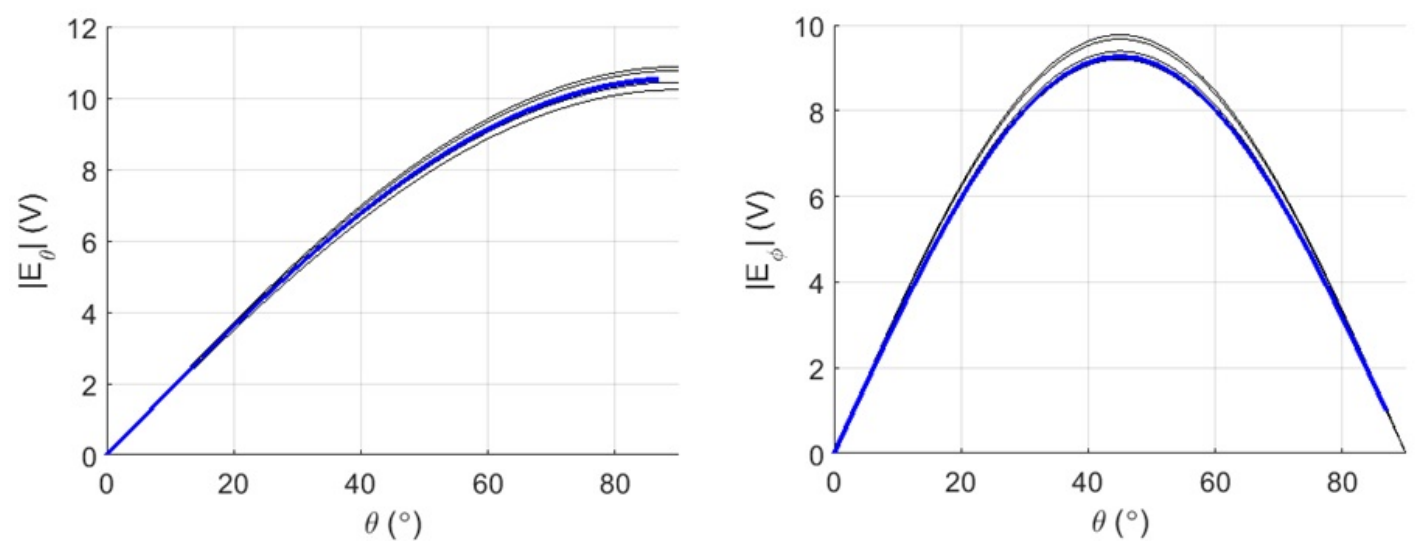

Figure 10. Error comparison of far-field electric field at $16 \mathrm{MHz}$ for Hertzian dipole sources.

The results in Figure 10 reveal similar error for the $\theta$-directed component of the field as in the $30-\mathrm{MHz}$ case. However, the error for the $\varphi$-directed component differs between the two frequencies. Since the error quantities are applied identically for both frequencies, a conclusion that can be made is that error does not necessarily propagate through the near-to-far field transformation identically for both polarizations. The difference may be a consequence of incomplete field information on the APR hemisphere. Unfortunately, capturing the low elevation field variation of a given $\varphi$-polarized antenna is difficult on the APR because the arch track does not allow for the probe antenna to be lowered below an elevation angle of $3^{\circ}$.

\section{MEASURED RESULTS}

To test the measurement technique, an AUT should be used that radiates both vertical and horizontal polarizations, which allows measurement verification of both polarizations. The dipoleloop antenna in Figure 11 was designed and built for this purpose. The dipole-loop antenna can be easily modeled in simulation to verify the near-field measurement and the far-field extraction.

A disadvantage of the dipole-loop antenna of Figure 11 is that, given the size of the loop portion, it is only an efficient radiator of the horizontal polarization at the upper end of the HF band. The antenna is not particularly well-matched at the lower end of the HF band. These two factors affect the gain pattern measurement accuracy. 


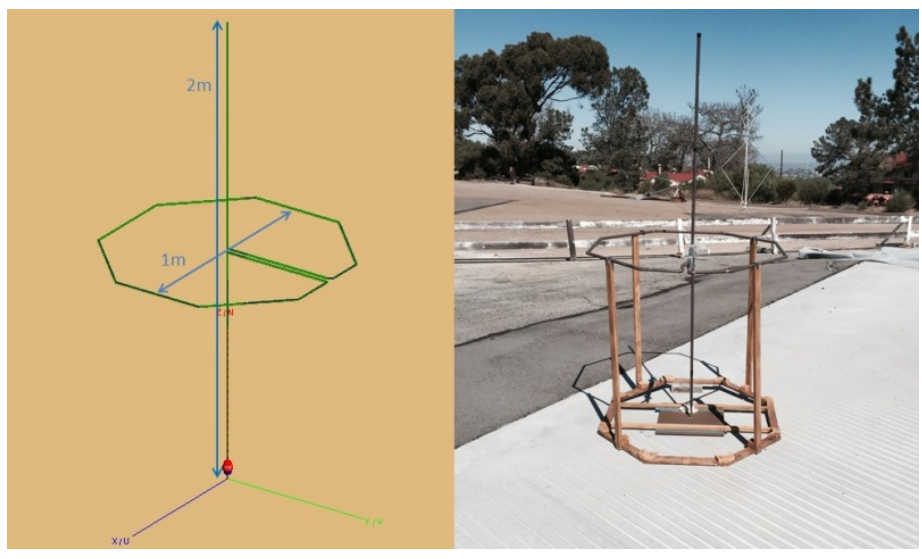

Figure 11. Dipole-loop antenna.

The plots in Figure 12 (plots provided in $\mathrm{dB}$ in Figure 13) compare the measured and simulated far-field electric field, both polarizations, of the dipole-loop antenna at $14 \mathrm{MHz}$. In this case, the radiated field of the dipole-loop antenna is only predominantly azimuthally invariant. The azimuthal variation is a consequence of the radially directed conductors that connect the dipole to the loop. Only the $\varphi=0^{\circ}$ cut is shown in Figure 12 and Figure 13, but a 3D plot of the far-field electric field (not shown) reveals azimuthal variation in the far-field $\theta$-component not captured by the near-to-far field extraction. Though measurement and simulation of the antenna were made across the entire HF band, Figure 12 only shows field results at $14 \mathrm{MHz}$ because these results succinctly reveal some of the relevant capabilities and limitations of the APR's use as a HF antenna measurement facility. Curves labeled "simulated data" indicate that the given field is computed using the near-to-far field extraction where the near-field data is taken from a simulation of the antenna. In the case of the simulated dipole-loop antenna, as shown in Figure 11, the near-field measurement points are made at the same locations on the APR hemisphere where the actual measurements are taken. In addition, no field values are taken for elevation angles below $3^{\circ}$ since the antenna track does not allow actual measurements to be taken below this angle. Curves labeled "measured data" indicate that the given field value is computed through the proposed measurement technique. Curves labeled "full simulation" indicate that the far-field is taken solely from simulation.

The measured $\theta$-directed electric field agrees with the full simulation field to nearly within the predicted error of the measurement method, which is $\pm 0.5 \mathrm{~dB}$. The far-field computed from a simulated near-field closely agrees with the simulated far-field, meaning the near-to-far field extraction method does a good job computing that component of the field. The plots of the $\varphi$-directed field suggest very different conclusions. The fully simulated results and the results extracted from simulated data appear to agree well for $\theta$ greater than $45^{\circ}$ (low elevation angles). The "simulated data” results are missing the same azimuthal variation of the far-field electric field. The difference between the two is likely a consequence of missing the $3^{\circ}$ of elevation measurements.

The $\varphi$-component of the field extracted from measurement appears to vary dramatically from the two simulated results. The variation is likely a consequence of low signal levels and measurement noise, which were not included as part of the error analysis presented in this report. At $14 \mathrm{MHz}, E_{\theta}$ is extracted accurately. But this accuracy degrades at lower frequencies as a consequence of the same low signal level and noise issues that were the presumed causes of error in the extracted $E_{\varphi}$ at $14 \mathrm{MHz}$. One simple solution to the low signal level problem is to include an amplifier between the AUT and the cable that connects it to the network analyzer. The cable is more than $150 \mathrm{ft}$ long, and therefore can significantly contribute to signal attenuation. 

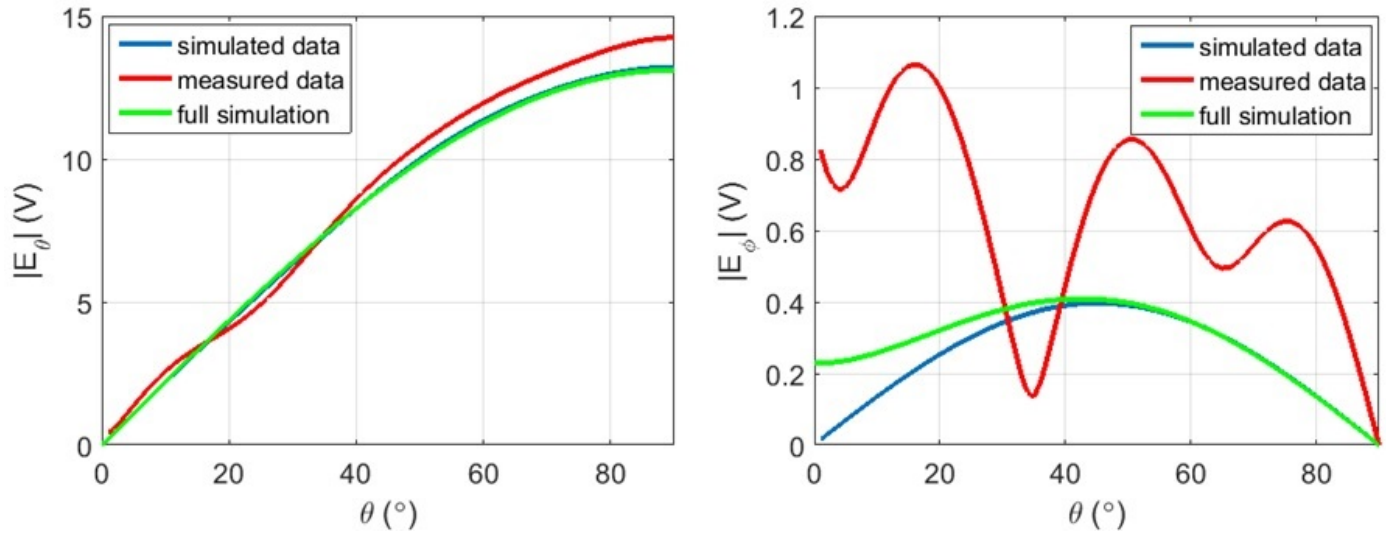

Figure 12. Far-field simulation and measurement comparison for dipole-loop antenna at $14 \mathrm{MHz}$.
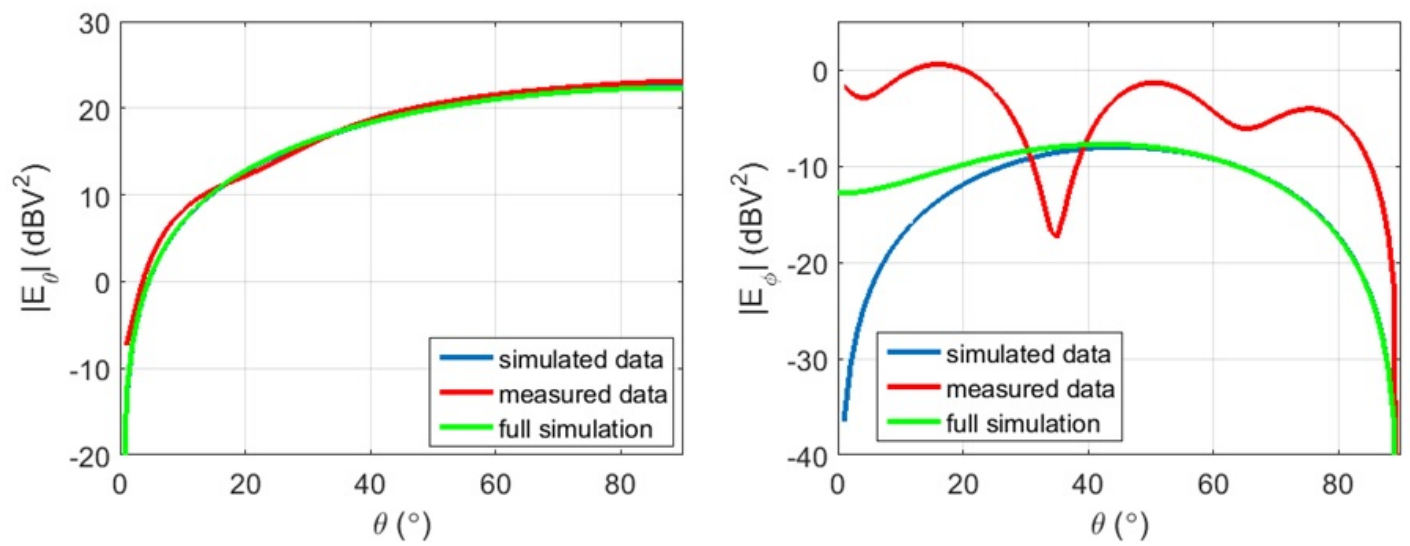

Figure 13. Far-field simulation and measurement comparison for dipole-loop antenna at $14 \mathrm{MHz}$ in $\mathrm{dB}$ (power reference). 


\section{CONCLUSION}

This report presents a description of a near-field measurement technique applied to the extraction of the far-field gain of HF radiators using the SSC Pacific APR. Estimates of the method's accuracy and the relevant reasoning behind such estimates were provided. Some of the limitations of the measurement technique and possible means of addressing these limitations were also described.

For vertically polarized HF antennas, the predicted gain error should be within $\pm 0.5 \mathrm{~dB}$. For horizontally polarized antennas, the measurement methodology is more susceptible to larger error. However, this error can be mitigated with sufficient signal amplification. We expect low elevation measurements above a conducting ground plane of a horizontally polarized electric field to be small. Such amplification may be necessary to elevate the signal above any measurement noise.

Horizontally polarized HF antennas are also more prone to measurement error on the APR due to the missing $3^{\circ}$ of elevation angle measurements that cannot be taken on it. The $\varphi$-directed near-field electric field varies dramatically as it approaches a value of zero at the horizon. Inaccurate characterization of the field variation appears to lead to inaccuracies in the extracted far-field quantities. Signal amplification may improve the accuracy of the far-field extraction. We will try this technique in future work.

We conclude that with sufficient AUT gain or barring and extra amplification in the measurement, this measurement technique is well-suited to characterize the absolute gain of vertically and horizontally polarized HF antennas. 


\section{REFERENCES}

1. D. Chen. 1989. Field and Wave Electromagnetics. Addison-Wesley Publishing Company, Reading, MA.

2. C. Balanis. 2005. Antenna Theory Analysis and Design. John Wiley \& Sons, New York, NY.

3. C. Balanis. 1989. Advanced Engineering Electromagnetics. John Wiley \& Sons, New York, NY.

4. J. Meloling and J. Allen. 2014. “A Near-to-Far Field Transformation Using Spherical Expansions.” Technical Report 2058. Space and Naval Warfare Systems Center Pacific (SSC Pacific), San Diego, CA.

5. T. Sarkar, P. Petre, A. Taaghol, and R. Harrington. 1997. “An Alternative Spherical Near Field to Far Field Transformation,” PIERs, vol. 16, pp. 269-284.

6. Altair Engineering. “FEKO Information.” Available online at https://www.feko.info/. Accessed December 2015. 


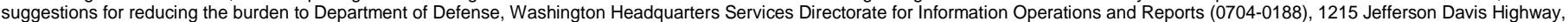

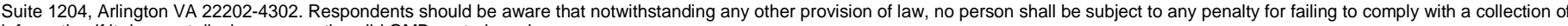

information if it does not display a currently valid OMB control number.

PLEASE DO NOT RETURN YOUR FORM TO THE ABOVE ADDRESS.

\begin{tabular}{l|l}
\hline 1. REPORT DATE $(D D-M M-Y Y Y Y)$ & 2. REPORT TYPE
\end{tabular}

December 2015

Final

\section{TITLE AND SUBTITLE}

\section{DATES COVERED (From - To)}

5a. CONTRACT NUMBER

Near-Field HF Antenna Pattern Measurement

Method Using an Antenna Pattern Range

5b. GRANT NUMBER

5c. PROGRAM ELEMENT NUMBER

\section{AUTHORS}

5d. PROJECT NUMBER

Ani Saripuram

Michael Daly

5e. TASK NUMBER

Michael Daly

5f. WORK UNIT NUMBER

7. PERFORMING ORGANIZATION NAME(S) AND ADDRESS(ES)

SSC Pacific, 53560 Hull Street, San Diego, CA 92152-5001

\section{PERFORMING ORGANIZATION REPORT NUMBER}

TR 3006

\section{SPONSORING/MONITORING AGENCY NAME(S) AND ADDRESS(ES)}

SSC Pacific

Naval Innovative Science and Engineering (NISE) Program

53560 Hull Street

San Diego. CA 92152-5001

\section{DISTRIBUTIONIAVAILABILITY STATEMENT}

Approved for public release.

\section{SUPPLEMENTARY NOTES}

This is work of the United States Government and therefore is not copyrighted. This work may be copied and disseminated without restriction.

\section{ABSTRACT}

A measurement method developed to extract the three-dimensional (3D) antenna gain patterns of high-frequency (HF) antennas is presented. The method employs a full hemispheric field probe measurement of an HF antenna on an outdoor antenna pattern range. A calibration method involving both simulation and measurement is used to transform antenna probe measurements into electric field quantities. The subsequent near-field electric field is extrapolated into the far field using an established near-field/farfield transformation method.

\section{SUBJECT TERMS}

Mission area: Communications

high-frequency antennas

near-field measurement

16. SECURITY CLASSIFICATION OF:

\begin{tabular}{|c|c|c|}
\hline a. REPORT & b. ABSTRACT & c. THIS PAGE \\
$\mathrm{U}$ & $\mathrm{U}$ & $\mathrm{U}$ \\
\hline
\end{tabular}

error estimate and correction

SSC Pacific Antenna Pattern Range
3D antenna gain patterns

calibration method

\section{LIMITATION OF ABSTRACT}

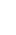

17. LIMITATION OF
ABSTRACT
$U$

\begin{tabular}{|l|l}
$\begin{array}{l}\text { 18. NUMBER } \\
\text { OF } \\
\text { PAGES }\end{array}$ & \\
\cline { 2 - 2 } &
\end{tabular}

19a. NAME OF RESPONSIBLE PERSON Michael Daly 19B. TELEPHONE NUMBER (Include area code) 


\section{INITIAL DISTRIBUTION}

$84300 \quad$ Library

$85300 \quad$ Archive/Stock

52250 A. Siripuram

$52250 \quad$ M. Daly

(1)

(1)

Defense Technical Information Center

Fort Belvoir, VA 22060-6218

(1) 
Approved for public release.

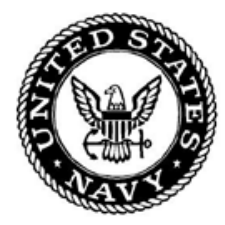

SSC Pacific San Diego, CA 92152-5001 